PROCEEDINGS OF THE

AMERICAN MATHEMATICAL SOCIETY

Volume 134, Number 9, September 2006, Pages 2481-2486

S 0002-9939(06)08649-7

Article electronically published on April 13, 2006

\title{
WITT KERNELS OF QUADRATIC FORMS FOR PURELY INSEPARABLE MULTIQUADRATIC EXTENSIONS IN CHARACTERISTIC 2
}

\author{
AHMED LAGHRIBI
}

(Communicated by Lance W. Small)

\begin{abstract}
The aim of this paper is to give a complete answer to the question of hyperbolicity of nonsingular quadratic forms over purely inseparable multiquadratic extensions in characteristic 2. This completes partial computations of Mammone and Moresi.
\end{abstract}

\section{INTRODUCTION}

Let $F$ denote a field of characteristic 2 , and let $W_{q}(F)$ denote the Witt group of nonsingular quadratic forms over $F \mathrm{~B} 2$. For a field extension $K / F$, consider the group homomorphism $W_{q}(F) \longrightarrow W_{q}(K)$ induced by the inclusion $F \subset K$. An important problem in the algebraic theory of quadratic forms is to compute the kernel $W_{q}(K / F)$ of this homomorphism.

Let us recall some examples of field extensions $K / F$ for which $W_{q}(K / F)$ was computed. It is well known that $W_{q}(K / F)$ is trivial for $K$ purely transcendental over $F$. Recently in L2] some general results were proven on $W_{q}(K / F)$ when $K$ is the function field of a quadratic form. On the other hand, very few results are known for $K / F$ algebraic of finite degree, and the case of multiquadratic extensions has aroused a lot of interest.

In characteristic 2, it is well known that a quadratic extension $K / F$ is either separable and thus is given by $K=F\left(\wp^{-1}(\alpha)\right)$ where $\wp: K \longrightarrow K$ is the homomorphism defined by $\wp(x)=x^{2}+x, x \in K$, or inseparable and thus is given by $K=F(\sqrt{\alpha})$ for some $\alpha \in F$.

For $\alpha_{1}, \alpha_{2} \in F$, we know the kernels for biquadratic extensions:

$$
\begin{gathered}
W_{q}\left(F\left(\wp^{-1}\left(\alpha_{1}\right), \wp^{-1}\left(\alpha_{2}\right)\right) / F\right)=W(F) \otimes\left[1, \alpha_{1}\right]+W(F) \otimes\left[1, \alpha_{2}\right], \\
W_{q}\left(F\left(\wp^{-1}\left(\alpha_{1}\right), \sqrt{\alpha_{2}}\right) / F\right)=W(F) \otimes\left[1, \alpha_{1}\right]+\left\langle 1, \alpha_{2}\right\rangle \otimes W_{q}(F), \\
W_{q}\left(F\left(\sqrt{\alpha_{1}}, \sqrt{\alpha_{2}}\right) / F\right)=\left\langle 1, \alpha_{1}\right\rangle \otimes W_{q}(F)+\left\langle 1, \alpha_{2}\right\rangle \otimes W_{q}(F),
\end{gathered}
$$

Received by the editors July 26, 2004 and, in revised form, January 7, 2005.

2000 Mathematics Subject Classification. Primary 11E04, $11 \mathrm{E} 81$.

Key words and phrases. Nonsingular quadratic forms, Witt groups, Witt kernels, differential forms.

The author was supported by the European research network HPRN-CT-2002-00287 "Algebraic $K$-Theory, Linear Algebraic Groups and Related Structures".

(C)2006 American Mathematical Society 
where $[\alpha, \beta]$ denotes the quadratic form $\alpha x^{2}+x y+\beta y^{2} ; W(F)$ is the Witt ring of regular symmetric bilinear forms over $F$; $\otimes$ is the action of $W(F)$ on $W_{q}(F)$ [B2]; and $\left\langle\alpha_{1}, \cdots, \alpha_{n}\right\rangle$ is the bilinear form $\sum_{i=1}^{n} \alpha_{i} x_{i} y_{i}$ for $\alpha_{1}, \cdots, \alpha_{n} \in F^{*}$.

The kernel (11) is due to Baeza [B2, Cor. 4.16, page 128]. The "mixed" kernel (22) is due to Ahmad [A. The kernel (3) is also due to Baeza in the case of quadratic extensions [B2, Lem. 4.3, page 182], and due to Mammone and Moresi in the case of biquadratic extensions [MM, Th. 2(i)]. Moreover, Mammone and Moresi proved that, in general, the kernel (11) does not generalize to separable triquadratic extensions [MM, Proposition 1], and the kernel (3) generalizes to inseparable multiquadratic extensions provided that the $W(F)$-submodule $I^{3} W_{q}(F)$ of $W_{q}(F)$ is trivial MM, Th. 2(ii)] (see below for the definition of this submodule).

Our aim in this paper is to prove that the kernel (3) generalizes to inseparable multiquadratic extensions without additional hypothesis on the ground field $F$. More precisely, we will prove the following theorem.

Theorem 1. Let $F$ be a field of characteristic 2 . Then for any scalars $\alpha_{1}, \cdots, \alpha_{n} \in$ $F(n \geq 1)$, we have

$$
W_{q}\left(F\left(\sqrt{\alpha_{1}}, \cdots, \sqrt{\alpha_{n}}\right) / F\right)=\sum_{i=1}^{n}\left\langle 1, \alpha_{i}\right\rangle \otimes W_{q}(F) .
$$

Let us make a comment on the situation when the characteristic is different from 2. Obviously, in this case a quadratic extension is of the form $F(\sqrt{\alpha})$ for some $\alpha \in F^{*}$. An analogous result of the kernel (1) for biquadratic extensions has been proved by Elman, Lam and Wadsworth ELW, Th. 2.10, page 137]. Still in this case, Elman, Lam, Tignol and Wadsworth constructed an example of a field for which the kernel (11) does not generalize to the case of triquadratic extensions ELTW, §5, page 1142].

For the proof of Theorem 1 we will begin with a generalization to purely inseparable multiquadratic extensions of a recent result by Aravire and Baeza $\mathrm{AB}$ concerning the behaviour of differential forms under inseparable quadratic extensions (Proposition 11). We also use a result by Kato [K] which establishes the connection between nonsingular quadratic forms and differential forms over $F$. We first reduce the proof of Theorem 10 to the case of a field which admits a finite 2-basis, and then give a proof in this case.

We suppose that the reader is familiar with the algebraic theory of quadratic forms in characteristic 2. For any unexplained notation and terminology we refer to [B2] or [HL], L1]. However, some definitions used here are taken from [HL], and some of them differ from those introduced in B2. For this reason, we fix again some rappels: A nonsingular quadratic form over $F$ is just an orthogonal sum of binary quadratic forms of type $[a, b]$ with $a, b \in F$. For $n \geq 1$, an $n$-fold Pfister form is a nonsingular quadratic form of type $\left\langle 1, a_{1}\right\rangle \otimes \cdots \otimes\left\langle 1, a_{n-1}\right\rangle \otimes\left[1, a_{n}\right]$ for $a_{1} \neq 0, \cdots, a_{n-1} \neq 0, a_{n} \in F$. We denote it by $\left\langle\left\langle a_{1}, \cdots, a_{n}\right]\right]$. Let $P_{n} F$ (resp. $G P_{n} F$ ) denote the set of $n$-fold Pfister forms up to isometry (resp. the set $\left.\left\{\alpha \pi \mid \alpha \in F^{*}, \pi \in P_{n} F\right\}\right)$. We denote by $I^{n} W_{q}(F)$ the $W(F)$-submodule of $W_{q}(F)$ generated by $G P_{n} F$.

Put $\Omega_{F}^{0}=F$. For $n \geq 1$, let $\Omega_{F}^{n}=\bigwedge^{n} \Omega_{F}^{1}$ denote the vector space of $n$-differential forms over $F$, where $\Omega_{F}^{1}$ is the $F$-vector space generated by symbols $d x, x \in F$, subject to the relations $d(x+y)=d x+d y$ and $d(x y)=x d y+y d x$ for $x, y \in F$. For $n \geq 1$, the map $d: F \longrightarrow \Omega_{F}^{1}: x \mapsto d x$, extends to a map $d: \Omega_{F}^{n} \longrightarrow \Omega_{F}^{n+1}$ called 
the differential operator and defined by

$$
d\left(x d x_{1} \wedge d x_{2} \wedge \cdots \wedge d x_{n}\right)=d x \wedge d x_{1} \wedge d x_{2} \wedge \cdots \wedge d x_{n} .
$$

For $\left\{b_{i} \mid i \in I\right\}$ a 2-basis of $F$, and after choosing an ordering on the set $I$, we get that $\left\{\frac{d b_{i_{1}}}{b_{i_{1}}} \wedge \cdots \wedge \frac{d b_{i_{n}}}{b_{i_{n}}} \mid i_{1}<\cdots<i_{n}\right\}$ is an $F$-basis of $\Omega_{F}^{n}$. There is a well-defined homomorphism $\wp_{n}: \Omega_{F}^{n} \longrightarrow \Omega_{F}^{n} / d \Omega_{F}^{n-1}$ given on generators by

$$
\wp_{n}\left(x \frac{d x_{1}}{x_{1}} \wedge \cdots \wedge \frac{d x_{n}}{x_{n}}\right)=\overline{\left(x^{2}-x\right) \frac{d x_{1}}{x_{1}} \wedge \cdots \wedge \frac{d x_{n}}{x_{n}}} .
$$

We denote by $\mathcal{H}^{n} F$ (resp. $\mathcal{Q}_{n}(F)$ ) the cokernel of $\wp_{n}$ (resp. the quotient $\left.I^{n} W_{q}(F) / I^{n+1} W_{q}(F)\right)$.

A fundamental result that we will use in this paper is due to Kato $\mathrm{K}$, and asserts that for any integer $n \geq 1$, there exists an isomorphism $k_{n}: \mathcal{Q}_{n}(F) \longrightarrow \mathcal{H}^{n-1} F$ given on generators by

$$
k_{n}\left(\overline{\left\langle\left\langle a_{1}, \cdots, a_{n}\right]\right]}\right)=\overline{a_{n} \frac{d a_{1}}{a_{1}} \wedge \cdots \wedge \frac{d a_{n-1}}{a_{n-1}} .}
$$

\section{A PRELIMINARY RESUlT}

To prepare the proof of Theorem 1, we begin with a generalization of $\mathrm{AB}$. Lem. 2.18] to the case of purely inseparable multiquadratic extensions:

Proposition 1. Let $F$ be a field of characteristic 2 and $\alpha_{1}, \cdots, \alpha_{m} \in F^{*}(m \geq 1)$. Then we have

$$
\operatorname{Ker}\left(\mathcal{H}^{n} F \longrightarrow \mathcal{H}^{n} F\left(\sqrt{\alpha_{1}}, \cdots, \sqrt{\alpha_{m}}\right)\right)=\sum_{i=1}^{m} \overline{\Omega_{F}^{n-1} \wedge d \alpha_{i}}
$$

Proof. Without loss of generality, we may suppose that $\left[F\left(\sqrt{\alpha_{1}}, \cdots, \sqrt{\alpha_{m}}\right): F\right]=$ $2^{m}$. We proceed by induction on $m$. The case $m=1$ has been proved by Aravire and Baeza [AB, Lem. 2.18]. Suppose $m \geq 2$ and that the proposition is true for any purely inseparable multiquadratic extension of degree $<2^{m}$ over a field of characteristic 2. Let $\omega \in \Omega_{F}^{n}$ be such that $\bar{\omega} \in \operatorname{Ker}\left(\mathcal{H}^{n} F \longrightarrow \mathcal{H}^{n} F\left(\sqrt{\alpha_{1}}, \cdots, \sqrt{\alpha_{m}}\right)\right)$. Since $\bar{\omega}_{F\left(\sqrt{\alpha_{1}}\right)} \in \operatorname{Ker}\left(\mathcal{H}^{n} F\left(\sqrt{\alpha_{1}}\right) \longrightarrow \mathcal{H}^{n} F\left(\sqrt{\alpha_{1}}, \cdots, \sqrt{\alpha_{m}}\right)\right)$, it follows from the induction hypothesis that

$$
\bar{\omega}_{F\left(\sqrt{\alpha_{1}}\right)}=\sum_{i=2}^{m} \overline{\omega_{i} \wedge d \alpha_{i}}
$$

for suitable $\omega_{2}, \cdots, \omega_{m} \in \Omega_{F\left(\sqrt{\alpha_{1}}\right)}^{n-1}$.

Claim: In equality (4), we may suppose $\omega_{2}, \cdots, \omega_{m}$ in $\Omega_{F}^{n-1}$, and thus again by the induction hypothesis applied to the field $F\left(\sqrt{\alpha_{1}}\right)$ the proof is complete.

Hence, it suffices to justify the claim. Since $\alpha_{1} \notin F^{* 2}$, we may choose a 2-basis $B \cup\left\{\alpha_{1}\right\}$ of $F$ such that $B \cup\left\{\sqrt{\alpha_{1}}\right\}$ is a 2-basis of $F\left(\sqrt{\alpha_{1}}\right)$.

Let us now fix $i \in\{2, \cdots, m\}$. We have $\omega_{i}=\sum_{\text {finite }} x_{j}^{i} \omega_{j}^{i}$ with $x_{j}^{i} \in F\left(\sqrt{\alpha_{1}}\right)^{*}$, and

$$
\omega_{j}^{i}=d c_{j_{1}}^{i} \wedge \cdots \wedge d c_{j_{n-1}}^{i}
$$

not zero with $c_{j_{1}}^{i}, \cdots, c_{j_{n-1}}^{i} \in B \cup\left\{\sqrt{\alpha_{1}}\right\}\left(j_{1}<\cdots<j_{n-1}\right)$.

- On the one hand, since equality (44) is taken modulo $\wp_{n}\left(\Omega_{F\left(\sqrt{\alpha_{1}}\right)}^{n}\right)$, we may suppose, after changing if necessary $x_{j}^{i} \omega_{j}^{i}$ by $\left(x_{j}^{i}\right)^{2} \omega_{j}^{i}$, that $x_{j}^{i} \in F^{*}$. 
- On the other hand, if there exists an index $j_{k}$ such that in the expression of $\omega_{j}^{i}$ (see above) we have $c_{j_{k}}^{i}=\sqrt{\alpha_{1}}$, say $j_{k}=j_{1}$, then $c_{j_{2}}^{i}, \cdots, c_{j_{n-1}}^{i} \in B$ since $\omega_{j}^{i}$ is not zero. But by using the differential operator $d$ we get

$$
\begin{aligned}
d\left[\left(x_{j}^{i} \sqrt{\alpha_{1}}\right) d c_{j_{2}}^{i} \wedge \cdots \wedge d c_{j_{n-1}}^{i} \wedge d \alpha_{i}\right]= & x_{j}^{i} \omega_{j}^{i} \wedge d \alpha_{i} \\
& +\sqrt{\alpha_{1}}\left(d x_{j}^{i} \wedge d c_{j_{2}}^{i} \wedge \cdots \wedge d c_{j_{n-1}}^{i} \wedge d \alpha_{i}\right) .
\end{aligned}
$$

Hence, in $\mathcal{H}^{n} F\left(\sqrt{\alpha_{1}}\right)$ we get

$$
\begin{aligned}
\overline{x_{j}^{i} \omega_{j}^{i} \wedge d \alpha_{i}} & \stackrel{(\mathbf{1})}{=} \overline{\left(\sqrt{\alpha_{1}}\right)\left(d x_{j}^{i} \wedge d c_{j_{2}}^{i} \wedge \cdots \wedge d c_{j_{n-1}}^{i} \wedge d \alpha_{i}\right)} \\
& \stackrel{(\mathbf{2})}{=} \overline{\alpha_{1}\left(d x_{j}^{i} \wedge d c_{j_{2}}^{i} \wedge \cdots \wedge d c_{j_{n-1}}^{i} \wedge d \alpha_{i}\right)}
\end{aligned}
$$

where for (1) (resp. for (2)) we proceed modulo $d \Omega_{F\left(\sqrt{\alpha_{1}}\right)}^{n-1}$ (resp. modulo $\left.\wp_{n}\left(\Omega_{F\left(\sqrt{\alpha_{1}}\right)}^{n}\right)\right)$. Hence the claim.

As a corollary of Kato's result (cited before) and Proposition 1, we get the following.

Corollary 1. With the same notation as in Proposition 1, we have

$$
\operatorname{Ker}\left(\mathcal{Q}_{n}(F) \longrightarrow \mathcal{Q}_{n}\left(F\left(\sqrt{\alpha_{1}}, \cdots, \sqrt{\alpha_{m}}\right)\right)\right)=\sum_{i=1}^{m} \overline{\left\langle 1, \alpha_{i}\right\rangle I^{n-1} W_{q}(F)} .
$$

Proof. Let $L=F\left(\sqrt{\alpha_{1}}, \cdots, \sqrt{\alpha_{m}}\right)$. We have a commutative diagram

$$
\begin{array}{ccc}
\mathcal{Q}_{n}(F) & \longrightarrow & \mathcal{Q}_{n}(L) \\
\downarrow & & \downarrow \\
\mathcal{H}^{n-1}(F) & \longrightarrow & \mathcal{H}^{n-1}(L)
\end{array}
$$

where the vertical arrows are given by the isomorphism $k_{n}\left(k_{n}\right.$ as before), and the horizontal arrows are induced by the inclusion $F \subset L$. Hence, $x \in \operatorname{Ker}\left(\mathcal{Q}_{n}(F) \longrightarrow\right.$ $\left.\mathcal{Q}_{n}(L)\right)$ implies that $k_{n}(x) \in \operatorname{Ker}\left(\mathcal{H}^{n-1} F \longrightarrow \mathcal{H}^{n-1} L\right)$. By Proposition 1 we deduce that $k_{n}(x) \in \sum_{i=1}^{m} \overline{\Omega_{F}^{n-2} \wedge d \alpha_{i}}$, and by the isomorphism $k_{n}$ it is clear that $x \in \sum_{i=1}^{m} \overline{\left\langle 1, \alpha_{i}\right\rangle I^{n-1} W_{q}(F)}$. Obviously,

$$
\sum_{i=1}^{m} \overline{\left\langle 1, \alpha_{i}\right\rangle I^{n-1} W_{q}(F)} \subset \operatorname{Ker}\left(\mathcal{Q}_{n}(F) \longrightarrow \mathcal{Q}_{n}(L)\right) .
$$

\section{Proof of Theorem 1}

Let $L=F\left(\sqrt{\alpha_{1}}, \cdots, \sqrt{\alpha_{n}}\right)$ and $\varphi \in W_{q}(L / F)$. Set $\varphi=\left[a_{1}, b_{1}\right] \perp \cdots \perp\left[a_{m}, b_{m}\right]$. We will give the proof in two steps.

1) Reduction to the case of a field with a finite 2-basis: Let $\mathbb{F}_{2}$ be the finite field with two elements and $F_{0}=\mathbb{F}_{2}\left(\alpha_{1}, \cdots, \alpha_{n} ; a_{1}, b_{1}, \cdots, a_{m}, b_{m}\right)$. The quadratic form $\varphi$ is defined over the subfield $F_{0}$ of $F$. Since $\varphi_{L}$ is hyperbolic, and after viewing $L^{2 m}$ as the underlying vector space of $\varphi_{L}$, there exist vectors $v_{1}, \cdots, v_{m} \in L^{2 m}$, linearly independent over $L$, such that $\varphi_{L}\left(v_{i}\right)=0$ and $B_{\varphi_{L}}\left(v_{i}, v_{j}\right)=0$ where $B_{\varphi_{L}}$ 
is the symmetric bilinear form associated to $\varphi_{L}$. Set $v_{i}=\left(w_{1}^{i}, \cdots, w_{2 m}^{i}\right)$ with $w_{1}^{i}, \cdots, w_{2 m}^{i} \in L(1 \leq i \leq m)$. For any $(i, k) \in\{1, \cdots, m\} \times\{1, \cdots, 2 m\}$, set

$$
w_{k}^{i}=\sum_{\text {finite }} c_{i_{1}, \cdots, i_{n}}^{i, k}\left(\sqrt{\alpha_{1}}\right)^{i_{1}} \ldots\left(\sqrt{\alpha_{n}}\right)^{i_{n}}
$$

with $i_{j} \in\{0,1\}$ and $c_{i_{1}, \cdots, i_{n}}^{i, k} \in F$. Now let

$$
K=F_{0}\left(c_{i_{1}, \cdots, i_{n}}^{i, k} \mid i=1, \cdots, m ; k=1, \cdots, 2 m\right)
$$

and

$$
K^{\prime}=K\left(\sqrt{\alpha_{1}}, \cdots, \sqrt{\alpha_{n}}\right) .
$$

Since $K^{\prime} \subset L$, the vectors $v_{1}, \cdots, v_{m}$ are linearly independent over $K^{\prime}$, and thus $\varphi_{K^{\prime}}$ is hyperbolic since $v_{1}, \cdots, v_{m} \in\left(K^{\prime}\right)^{2 m}$. Clearly, $K$ has a finite 2-basis since it is finitely generated over $\mathbb{F}_{2}$. Moreover, we may suppose that $\varphi$ is not hyperbolic over $F$, and thus it is not hyperbolic over $K$. Since an element of $\sum_{i=1}^{n}\left\langle 1, \alpha_{i}\right\rangle W_{q}(K)$ can be considered as an element of $\sum_{i=1}^{n}\left\langle 1, \alpha_{i}\right\rangle W_{q}(F)$ after extending scalars to $F$, we see that the proof can be reduced to the case of a field with a finite 2-basis.

2) The case where $F$ has a finite 2-basis: If $n_{0}$ is the number of elements of a 2-basis, we deduce that the space $\Omega_{F}^{i}$ is trivial for $i>n_{0}$. By Kato's result and the Hauptsatz of Arason and Pfister [B1, Satz 4.1], the group $I^{i} W_{q}(F)$ is also trivial for $i>n_{0}+1$. Let $r \geq 1$ be such that $\varphi \in I^{r} W_{q}(F)$. Since $\varphi_{L}$ is hyperbolic, we deduce that $\bar{\varphi} \in \operatorname{Ker}\left(\mathcal{Q}_{r}(F) \longrightarrow \mathcal{Q}_{r}(L)\right)$. It follows from Corollary 1 that $\bar{\varphi} \in \sum_{i=1}^{n} \overline{\left\langle 1, \alpha_{i}\right\rangle I^{r-1} W_{q}(F)}$. Let $\rho_{1}, \cdots, \rho_{n} \in I^{r-1} W_{q}(F)$ be such that

$$
\varphi \perp \sum_{i=1}^{n}\left\langle 1, \alpha_{i}\right\rangle \otimes \rho_{i} \in I^{r+1} W_{q}(F) .
$$

It is clear that the form $\varphi^{\prime}:=\varphi \perp \sum_{i=1}^{n}\left\langle 1, \alpha_{i}\right\rangle \otimes \rho_{i}$ is hyperbolic over $L$. If $\varphi^{\prime}$ is hyperbolic over $F$, then we are done. If not, we reproduce the same argument as above for $\varphi^{\prime}$ to get $\varphi^{\prime} \perp \sum_{i=1}^{n}\left\langle 1, \alpha_{i}\right\rangle \otimes \rho_{i}^{\prime} \in I^{r+2} W_{q}(F)$ for some quadratic forms $\rho_{1}^{\prime}, \cdots, \rho_{n}^{\prime} \in I^{r} W_{q}(F)$, i.e., $\varphi^{\prime \prime}:=\varphi \perp \sum_{i=1}^{n}\left\langle 1, \alpha_{i}\right\rangle \otimes\left(\rho_{i}^{\prime} \perp \rho_{i}\right) \in I^{r+2} W_{q}(F)$. If $\varphi^{\prime \prime}$ is hyperbolic over $F$, then $\varphi \in \sum_{i=1}^{n}\left\langle 1, \alpha_{i}\right\rangle \otimes W_{q}(F)$ and we are done. If not, we continue the process in order to get a quadratic form $\varphi \perp \sum_{i=1}^{n}\left\langle 1, \alpha_{i}\right\rangle \otimes \delta_{i} \in I^{k} W_{q}(F)$ for some $\delta_{1}, \cdots, \delta_{n} \in W_{q}(F)$ and $k>n_{0}+1$, and thus to get a hyperbolic quadratic form.

\section{A Question}

Before we formulate a general question, let us recall that the function field of a symmetric bilinear form $B$, denoted by $F(B)$, is the function field of the quadratic form $\widetilde{B}$ defined by $\widetilde{B}(v)=B(v, v)$ for $v \in V$, where $V$ denotes the underlying vector space of $B$ ( $\widetilde{B}$ is uniquely determined by the isometry class of $B)$. In particular, the function field of the bilinear form $\langle 1, \alpha\rangle, \alpha \in F^{*}$, is the field $F(x)(\sqrt{\alpha})$ for $x$ a 
variable over $F$. Hence, Theorem 1 describes the kernels $W_{q}\left(F\left(B_{1}\right) \cdots\left(B_{n}\right) / F\right)$ for $B_{i}=\left\langle 1, \alpha_{i}\right\rangle(1 \leq i \leq n)$. In view of this it is natural to ask the following question.

Question 1. Let $F$ be a field of characteristic 2 , and let $B_{1}, \cdots, B_{n}$ be symmetric bilinear Pfister forms of dimension $\geq 2(n \geq 1)$. Is it true that the kernel $W_{q}\left(F\left(B_{1}\right) \cdots\left(B_{n}\right) / F\right)$ equals $B_{1} \otimes W_{q}(F)+\cdots+B_{n} \otimes W_{q}(F)$ ?

\section{ACKNOWLEDGMENTS}

I am grateful to Pasquale Mammone who drew my attention on the problem studied in this paper, and for some discussions that I have had with him during the preparation of this work. I also thank Bruno Kahn for some comments on an earlier version of this work.

\section{REFERENCES}

[A] H. Ahmad, Witt kernels of bi-quadratic extensions in characteristic 2, Bull. Austral. Math. Soc. 69 (2004), 433-440. MR2066661 (2005g:11058a)

[AB] R. Aravire, R. Baeza, The behavior of quadratic and differential forms under function field extensions in characteristic two, J. Algebra 259 (2003), 361-414. MR.1955526 (2003k:11060)

[B1] R. Baeza, Ein Teilformensatz für quadratische Formen in Charakteristik 2, Math. Z. 135 (1974), 175-184. MR0337765 (49:2534)

[B2] R. Baeza, Quadratic forms over semilocal rings, Lect. Notes Math. vol. 655, Berlin, Heidelberg, New York, Springer 1978. MR0491773 (58:10972)

[ELTW] R. Elman, T. Y. Lam, J.-P. Tignol, A. Wadsworth, Witt rings and Brauer groups under multiquadratic extensions I, Amer. J. Math. 105 (1983), 1119-1170. MR0714772 (85i:11032)

[ELW] R. Elman, T. Y. Lam, A. Wadsworth, Quadratic forms under multiquadratic extensions, Indag. Math. 42 (1980), 131-145. MR0577569 (81j:10028)

[HL] D. W. Hoffmann, A. Laghribi, Quadratic forms and Pfister neighbors in characteristic 2, Trans. Amer. Math. Soc. 356 (2004), 4019-4053. MR2058517 (2005e:11041)

[K] K. Kato, Symmetric bilinear forms, quadratic forms and Milnor K-theory in characteristic 2, Inventiones Math. 66 (1982), 493-510. MR0662605 (83i:10027)

[L1] A. Laghribi, Certaines formes quadratiques de dimension au plus 6 et corps des fonctions en caractéristique 2, Israel J. Math. 129 (2002), 317-361. MR.1910948(2003f:11047)

[L2] A. Laghribi, Witt kernels of function field extensions in characteristic 2, J. Pure Appl. Algebra 199 (2005), 167-182. MR2134299 (2006c:11041)

$[\mathrm{MM}] \quad \mathrm{P}$. Mammone, R. Moresi, Formes quadratiques, algèbres à division et extensions multiquadratiques inséparables, Bull. Belg. Math. Soc. 2 (1995), 311-319. MR.1338463 (96j:11052)

Laboratoire de Mathématiques de Lens EA 2462, Faculté des Sciences Jean Perrin, Rue Jean Souvraz - SP18, F-62307 Lens, France

E-mail address: laghribi@euler.univ-artois.fr 